\title{
Article \\ Augmented Sensitivity of At-Home Rapid SARS-CoV-2 Antigen Test (RAT) Kits with Computer Vision
}

\author{
Kim Miikki ${ }^{1}$, Lars Miikki ${ }^{2}$, Jenny Wiklund ${ }^{3,4}$ and Alp Karakoç ${ }^{4, *}$
}

\author{
Aalto University, School of Chemical Engineering, Espoo, Finland; kim.miikki@aalto.fi \\ Aalto University, School of Science, Espoo, Finland; lars.miikki@aalto.fi \\ Aalto University, Department of Bioproducts and Biosystems, Espoo, Finland; jenny.wiklund@aalto.fi \\ Aalto University, Department of Communications and Networking, Espoo, Finland; alp.karakoc@aalto.fi \\ * Correspondence: alp.karakoc@aalto.fi
}

\begin{abstract}
At-home rapid antigen test (RAT) kits for severe acute respiratory syndrome coronavirus 2 (SARS-CoV-2) are valuable public health tools during the present coronavirus disease (COVID-19) pandemic. They provide fast identification of coronavirus infection, which can help to reduce the transmission rates and burden on the healthcare system. However, they have lower sensitivity when compared with the reverse transcription polymerase chain reaction (RT-PCR) tests. One of the reasons for the lower sensitivity is due to the RAT color indicators being indistinct or invisible to the naked eye after the measurements. For this reason, we propose a systematic approach, through which we investigated anonymously provided at-home RAT kit results by using our in-house open source image processing scripts developed for affordable Raspberry Pi computer and Raspberry Pi HQ camera systems (available at https://github.com/kmiikki/ratcv). Therefore, we aimed at minimizing the human-related analysis errors for such kits. We believe that our framework can contribute to reduced the delayed quarantines of infected individuals and spreading of the current infectious disease.
\end{abstract}

Keywords: SARS-CoV-2; rapid antigen test; RT-PCR test; COVID-19; image processing; Raspberry Pi

\section{Introduction}

Since being declared as a global pandemic, severe acute respiratory syndrome coronavirus 2 (SARS-CoV-2) has caused dramatic public health challenges all over the world [1-3]. In order to overcome the pandemic and its consequences, various strategies including lockdowns, isolations and preventive acts (widespread use of face masks, sanitizers, and social distancing) have been implemented worldwide [4-6]. Although these strategies have been effectively implemented, they are expected to result in economical and social challenges in the following years due to reduced labor and disruptions in supply-chains $[7,8]$. Therefore, in order to minimize the spreading of SARS-CoV-2 and constantly maintain it at a baseline level, medical community has been working on rapid, easy-to-use and affordable testing $[9,10]$. For this goal, several assays have been developed in order to identify the infected people, who may have almost no symptoms (asymptomatic), very mild, or distinct symptoms [11-13]. Time span of sampling and sensitivity of the assays are crucial, especially, for quarantine and contact tracing at early stages [14,15]. Therefore, unprecedented global infectious diseases could be curtailed.

Among the assays, the sensitivity and specificity (referring to the probabilities of positive and negative tests, respectively) of reverse transcription polymerase chain reaction (RT-PCR) tests are undeniable [16-18]. RT-PCR tests are accepted as the 'Gold standard tests', which require extraction of viral RNA as well as stationary instrumentation for nucleic acid amplification and detection [19]. Diagnostic laboratories routinely perform RT-PCR tests as being considered the most reliable methodology for testing of cases and contacts [20]. However, requirements for designated laboratories and equipment, specialized staff, and several hours of turnaround time for RT-PCR tests, in turn, may result in delayed diagnosis and quarantine of the infected individuals, which further causes spreading of the disease [21]. In addition, the cost of RT-PCR tests, and current global demand 
and procurement challenges also result in jeopardizing the health care infrastructure and management [22].

Despite their lower sensitivity compared to RT-PCR tests, rapid SARS-CoV-2 antigen test (RAT) kits, which are immunochromatographic assays, can be affordable and timely solution for identification and quarantine of infected individuals with minimal need of training and equipment $[23,24]$. RAT kits are intended for the comparative detection of nucleocapsid protein from SARS-CoV-2 in nasal swab specimens, for which the samples have to be collected within 7 days of coronavirus disease (COVID-19) symptom onset [25]. RATs detect the presence of a specific viral antigen, which implies current viral infection. They are authorized to be performed on nasopharyngeal or nasal swab specimens placed directly into the assay's extraction buffer or reagent. The currently authorized tests include point-of-care, laboratory-based, and at-home (self-tests), and are applicable to people of any age [26]. The recently developed RAT kits offer multiple benefits in comparison to RT-PCR tests for the detection of SARS-CoV-2. They are simpler than RT-PCR tests because they do not require specially trained laboratory staff or a specialized laboratory. Results are normally generated in 10 to 30 minutes after the start of the analysis, and at low cost [27]. Therefore, especially, at-home RAT kits have been increasingly available for the public use on the market shelves. Several hundreds of CE marked RATs have been recently included in the 'COVID-19 In Vitro Diagnostic Devices and Test Methods Database' of the European Commission [28].

Despite their pros, publicly available off-the-shelf at-home RAT kits quite often produce lower sensitivity compared to the standard RT-PCR tests (while their specificity is generally reported to be high) [29]. Unless there are significant symptoms, follow-up RTPCR tests or RAT are ignored by most of the individuals, which are related to the increasing costs of repetitive testing (thus, financial burdens), extensive reservation queues for official testing and high demand-low level of availability of at-home RAT kits in the market. As a result of initial false negatives (due to lower RAT sensitivity) and challenges with follow-up testing, the delays for preventive action and quarantine are inevitable, which easily results in infectious disease spreading and places heavy burden on the healthcare infrastructure.

As illustrated in Figure 1, in order to ensure and enhance the observed sensitivity of the at-home RAT kits, we assessed anonymous RAT results and developed a computer vision framework by using open-source image processing libraries developed for highly affordable Raspberry Pi computer and Raspberry Pi HQ camera system. The findings and provided libraries (at https:/ / github.com/kmiikki/ratcv) can be immediately integrated with and augmented the sensitivity of the RAT kit results, which can not be distinguished with the naked eye. 

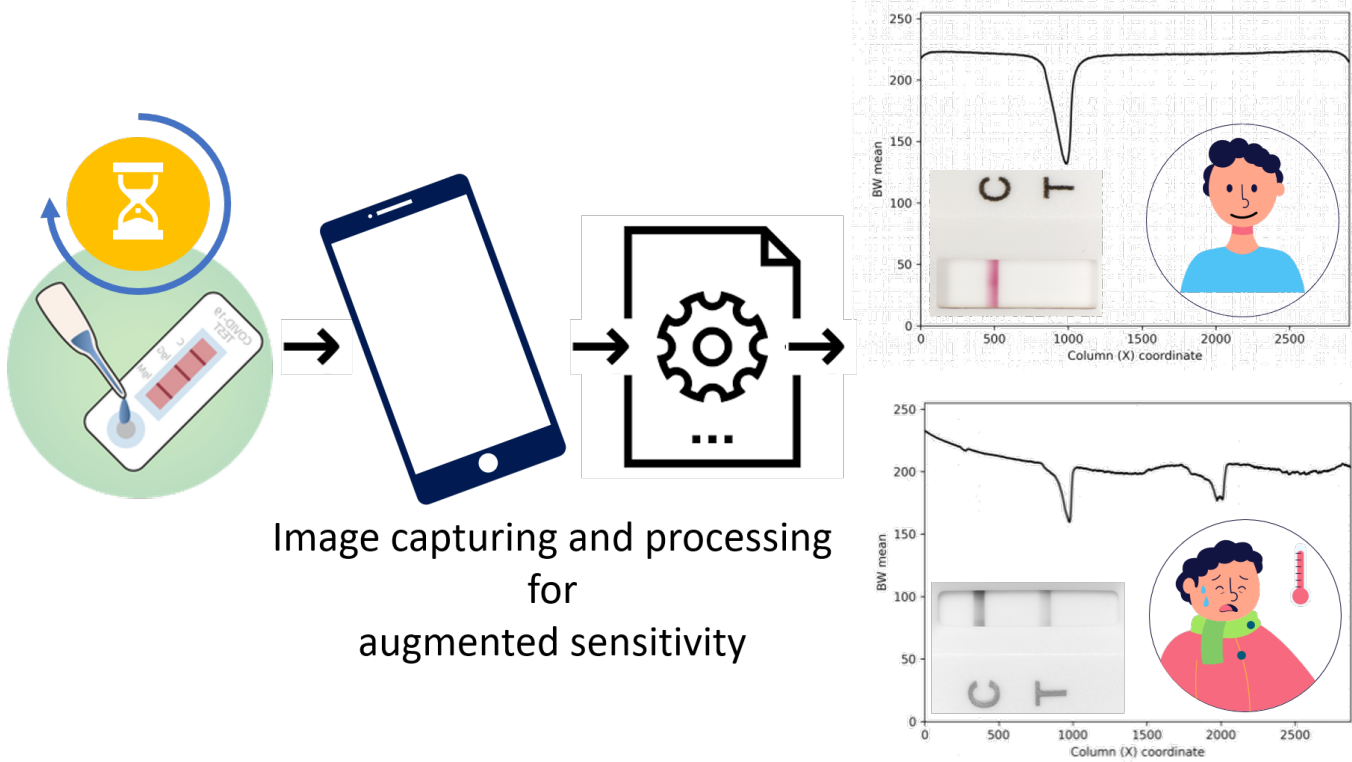

Figure 1. The present framework for augmented sensitivity of at-home rapid antigen test (RAT) kits with affordable hardware and open-source scripts and libraries.

\section{Materials and Methods}

\subsection{Foundations of the evaluated assays}

Lateral flow assays are the technology behind low-cost, simple, rapid and portable detection devices popular in biomedicine, agriculture, food and environmental sciences [30]. Before the COVID-19 pandemic, the most commonly known type of lateral flow rapid tests was the pregnancy test.

The immunochromatography devices used in this study are lateral flow immunoassays (LFIA) for the qualitative detection of specific antigens (nucleocapsid protein, nucleoprotein) of SARS-CoV-2 present in the human nasopharynx. LFIA is the main type of commercially available RATs for diagnostic self-testing for SARS-CoV-2 infection. A positive result implies a current viral infection.

The principle of LFIA is based on the movement of a liquid sample though a polymeric strip with attached molecules that interact with the analyte, providing a signal that can be visually detected. As shown in Figure 2, a typical lateral flow test strip consists of overlapping membranes that are mounted on a backing card. The test strip is typically composed of the following elements: sample pad, conjugate release pad, membrane with immobilized antibodies and adsorbent pad. The components of the strip are usually fixed to an inert backing material for better stability and handling [30]. In RAT kits the strip is in general packaged in a small cartridge that protects its components and makes the test very simple for anyone to perform. The result is visible for the naked eye.

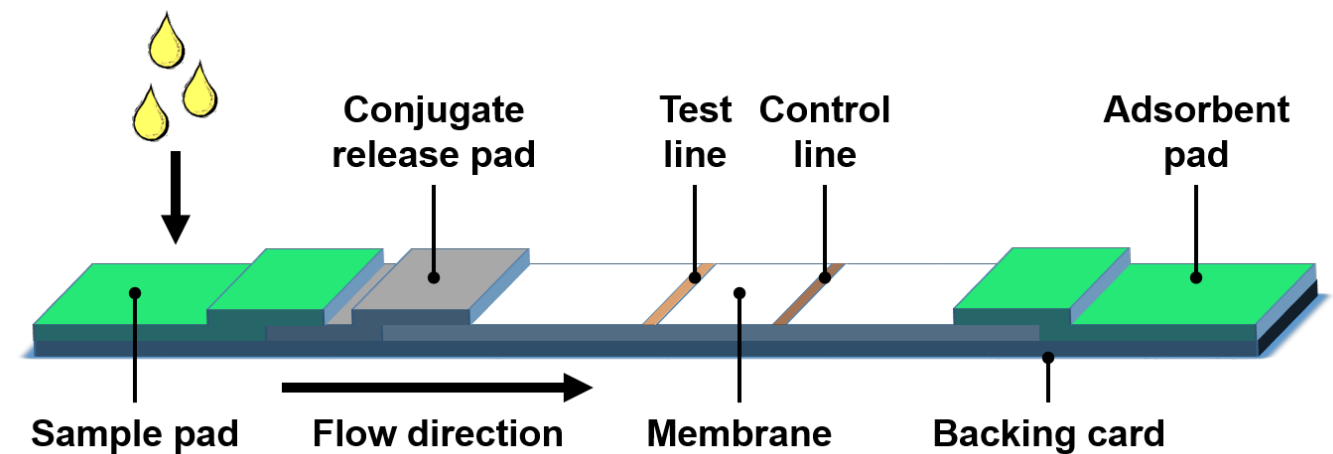

Figure 2. Lateral flow immunoassay (LFIA) test strip configuration. 
LFIA devices typically apply immunoassay technology using nitrocellulose membrane, colored nanoparticles (or labels), and antibodies, to produce results. An antibody, also known as an immunoglobulin, is a large, Y-shaped protein, which recognizes a unique (macro)molecule of the pathogen, called an antigen [31].

To start the assay, the sample is applied on the sample pad, which may be impregnated with buffer salts and surfactants that make the sample suitable for interaction with the detection system. The function of this pad is to transport the sample to other components of the device. The sample then migrates through the conjugate release pad, which typically contains labeled antibodies that are specific to the target analyte. The antibodies are usually labeled by nano colloid gold particles. If the target is present, the labeled antibodies will bind to the target and form a complex. The sample, containing the complex, then migrates along the strip into the detection zone, which is a porous membrane (usually nitrocellulose) with specific biological components immobilized in lines. As the sample moves along the membrane the binding reagents attached to test line will bind to the complex containing the target analyte bound to the labeled antibody. A colored line will form and the density of the line will vary depending on the quantity of the target present. Visual control line indicates that the test has run correctly. The read-out, represented by the lines appearing, can be assessed by eye or computer vision for augmented sensitivity as proposed in the present study. The liquid flows across the device because of the capillary force of the strip material and, to maintain this movement, an absorbent pad is attached at the end of the strip. The role of this pad is to wick the excess reagents and prevent backflow of the liquid [30].

\subsection{Sampling and color analysis}

Total amount of people who participated in COVID-19 in vitro antigen testing was 9, and the tests used were: Boson Biotech Rapid SARS-CoV-2 Antigen Test Card (cases 1 and 2), Alltest SARS-CoV-2 Antigen Rapid Test (Nasal Swab) (cases 3 and 4) and Deepblue COVID-19 (SARS-CoV-2). According to the manufacturers, all these tests met the minimum recommendations for Rapid antigen detection tests, given by the European Center for Disease Prevention and Control: $\geq 90 \%$ sensitivity and $>98 \%$ specificity [32].

The images and videos for the RGB analysis were captured with a Raspberry Pi based system [33]. The optical setup for imaging RAT windows is shown in Figure 3. A $35 \mathrm{~mm}$ Waveshare telephoto lens for Pi was used with three C-CS adapters to get a minimum focusing distance close-enough for macro photography. The test cassette was illuminated with three Manfrotto Lumimuse 8 LED lights, and the colors were calibrated by using a gray card (Lastolite Ezybalance).

Color analysis used in this study, is based on directional color profiling by red, green and blue colors. These colors are marked in all figures as R, G and B. The mean of the three colors is interpreted as gray, and noted here as black and white (BW). Color profiling is normally done in horizontal or vertical directions. Each row or column of pixels are read, its means of $R, G$ and $B$ are calculated, and these values represents the color values for the current coordinate ( $\mathrm{x}$ or $\mathrm{y})$. The eluent in a chromatographic test moves in one direction, the test lines are aligned perpendicular to the fluid direction, therefore color profiling is only needed in one direction which has been chosen here as horizontal (x) direction. 


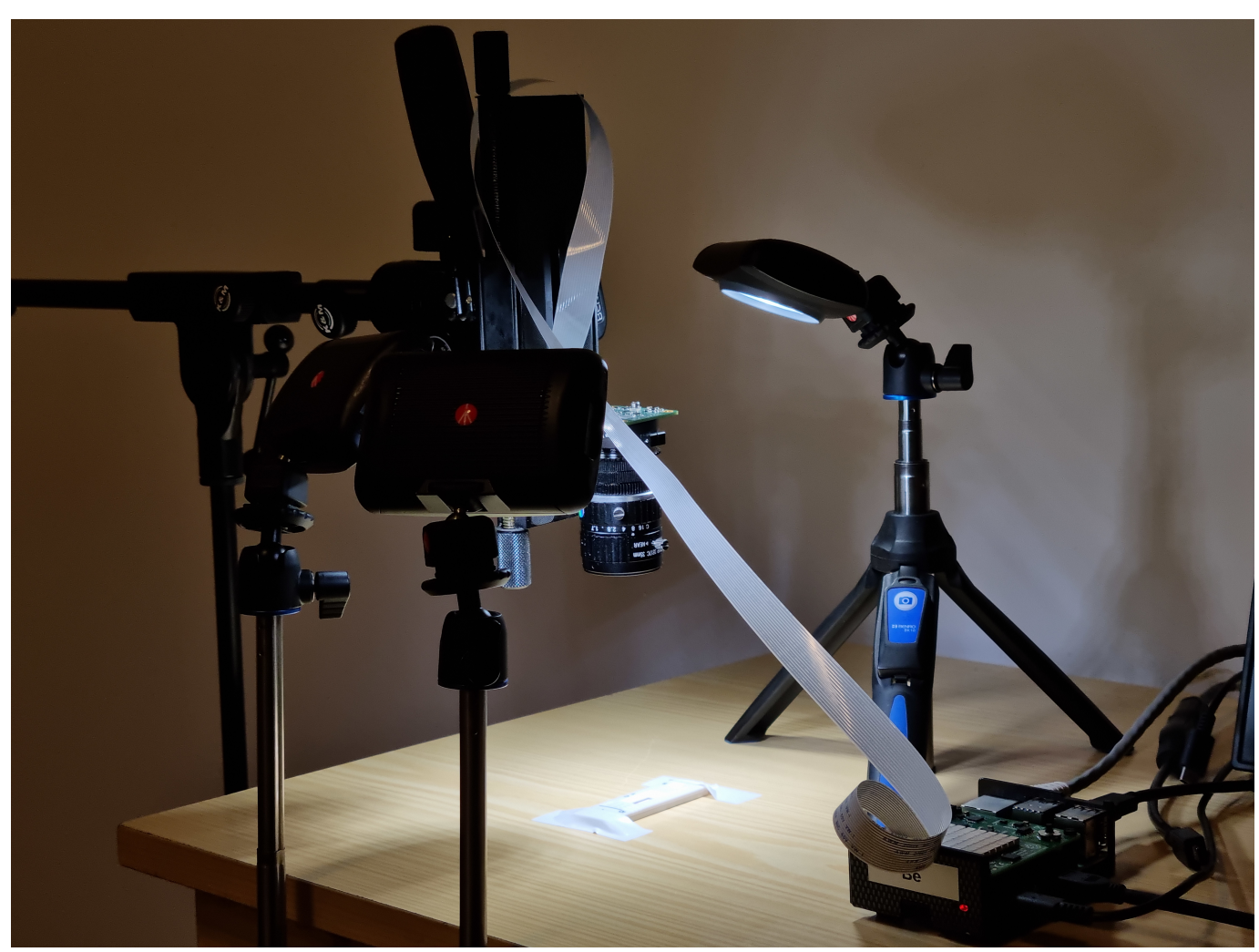

Figure 3. A Raspberry Pi based photo system for macro imaging coronavirus disease (COVID-19) RAT windows.

All captured images were processed and analyzed with Python scripts [34]. Before capturing any pictures, the Raspberry Pi camera sensor red and blue gains were calibrated by using the mapgains.py. Thereafter manual white balance, with the acquired gain values, were used with a constant shutter speed. In order to perform a decent directional color analysis, the image should be straight and only the test windows should be analyzed. The corresponding image operations are rotate and crop. The image composition should be composed properly, so there should not be any need to rotate the images. The directional color analysis can be performed with rgbxy.py, the peak properties can be examined with rgbxy-peaks.py, and finally the internal ratios of red, green and blue can be inspected by executing rgbxy-iratios.py.

\section{Results}

\subsection{Detection of simulated weak test lines}

The white part of a negative test, which is described in the next subsection, was used as a template for simulated weak test lines. These were created with testlines.py script. Red, green and blue ratios were acquired from a positive test line which were analyzed with rgb-peaks.py peak find utility. The color ratios for the simulated lines are $\mathrm{R}: \mathrm{G}: \mathrm{B}=$ $0.21: 0.43: 0.36$, where $R$ is a peak and G, $B$ are valleys. The line width is 40 , including a 35 pixels wide gradual fading on the right side, the distance between two lines is 50 pixels wide, and the maximum strengthening per line is 2.0. The following command generates the Figure 4, when the name of the template is test_area.png: testlines.py test_area.png -w 40 -d 50 -right -r -dec -grad 35 -rf 0.21 -gf 0.43 -bf 0.36 -s 2. 
Figure 4. Weak test lines created with testlines.py script.

(a)

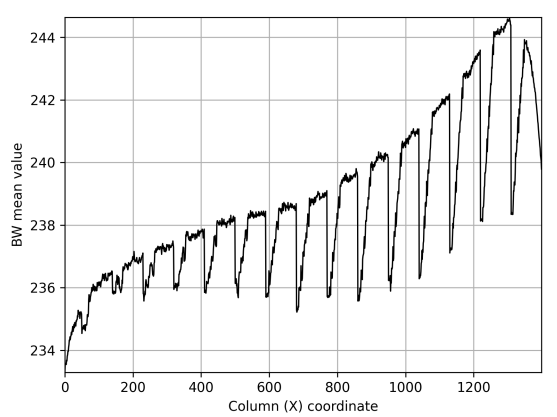

(b)

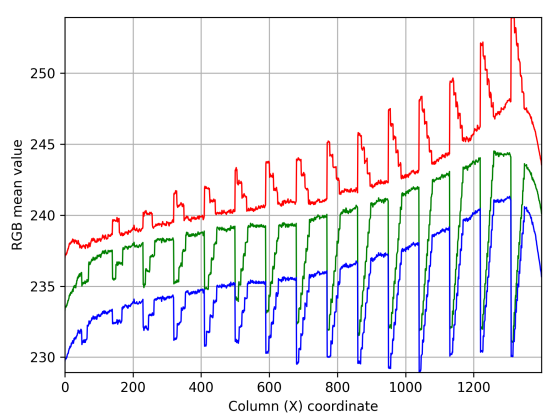

Figure 5. Horizontal color profiles of the test lines image: (a) BW, (b) RGB.

A directional color analysis of the created test lines was performed, and the horizontal BW (mean of R, G and B channels) and RGB profiles are shown in Figure 5. The weakest lines are barely seen in the BW profile, but they are more visible in the RGB profile. However, when dividing colors with each other, or with the means of two colors, the color differences can often be enhanced, especially if any of the color peaks point in the opposite direction. This can be seen in Figure 6, where the R represent a color peak and the G and B color valleys.

(a)

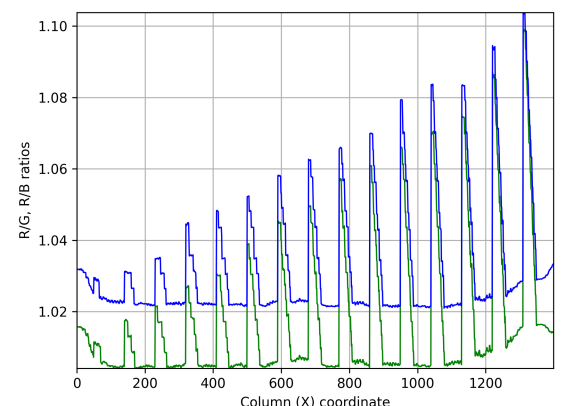

Figure 6. Horizontal color ratios of the created test lines: (a) R/G and R/B, (b) R/GB, G/RB and $\mathrm{B} / \mathrm{RG}$.

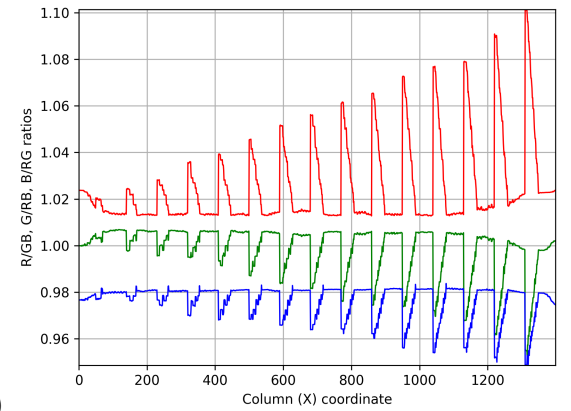

(b) 


\subsection{Case 1 Negative (reference case)}

A negative COVID-19 RAT was performed as a reference, by analyzing only the extraction buffer without a biological specimen. The control line (C line) appeared in the test window, which can be seen in Figure 7. In the Figure 8, only one valley is detected at the same $\mathrm{x}$ coordinate range as the $\mathrm{C}$ line, as expected. The red color weakening is lesser than blue and green, hence the $\mathrm{R} / \mathrm{G}$ and $\mathrm{R} / \mathrm{B}$ ratios give strong peaks, and the $\mathrm{C}$ line has a red hue. The RAT was done correctly, and the result is negative.

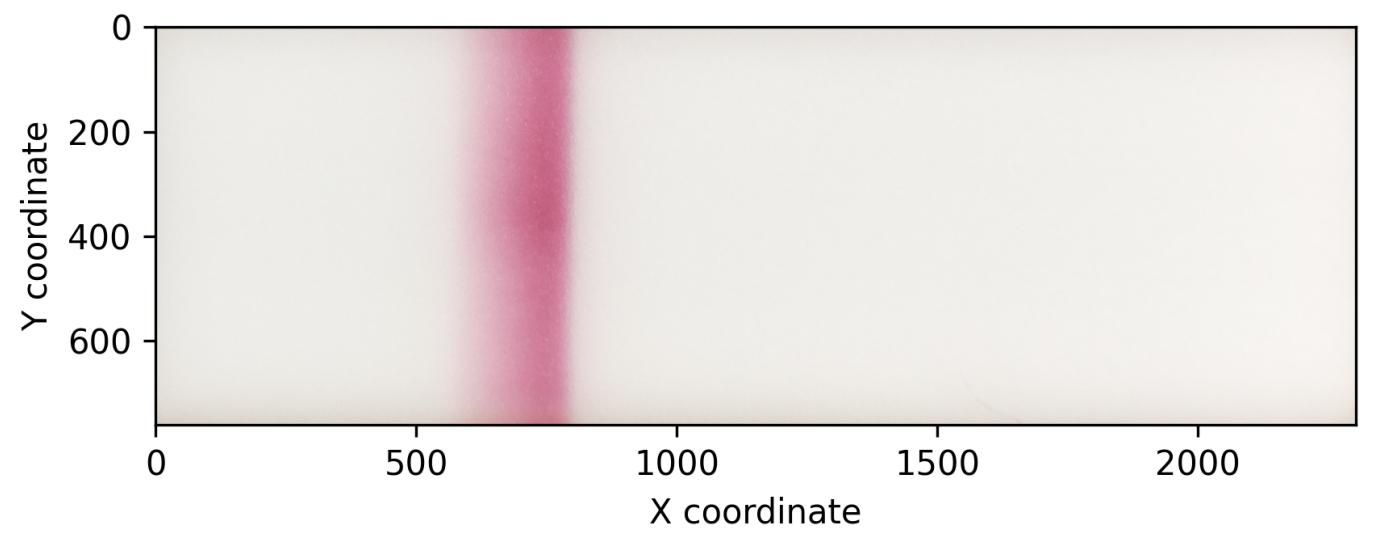

Figure 7. Negative RAT result of the extraction buffer liquid: only control line (C line) is visible.

(a)

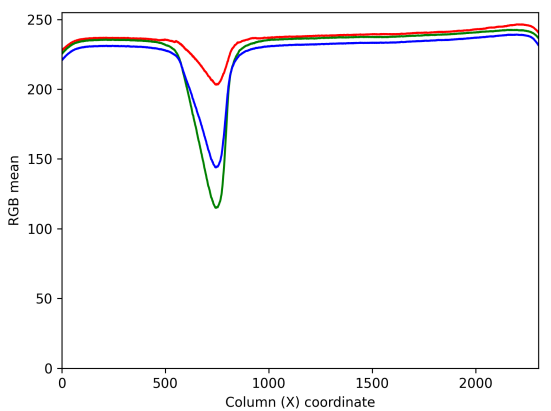

(b)

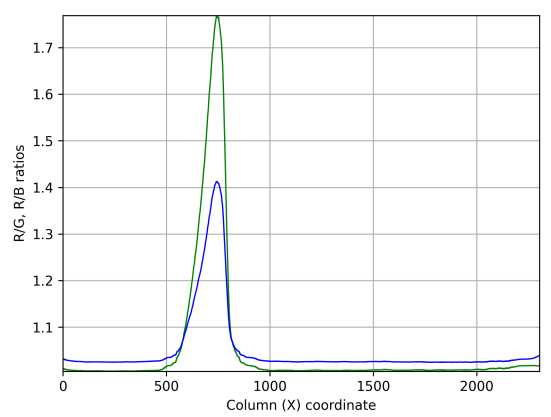

Figure 8. (a) RGB color profile in horizontal direction, (b) R/G and R/B ratios profile in horizontal direction. 


\subsection{Case 2 False negative - very weak positive}

In this case, the picture was acquired in auto white balance mode with a smartphone (Nokia 8 Sirocco) after the RAT, and analyzed with a Raspberry Pi computer. The image was cropped and a directional RGB analysis was performed in $\mathrm{x}$ direction. The result was negative with the naked eye, as well with the basic color analysis (Figure 10 (a)). However, as seen in Figure 10 (b), a very weak signal can be detected at $x=350-400$, if the red channel values are divided with green or blue color values. An official RT-PCR test was performed next day, and the result was positive.

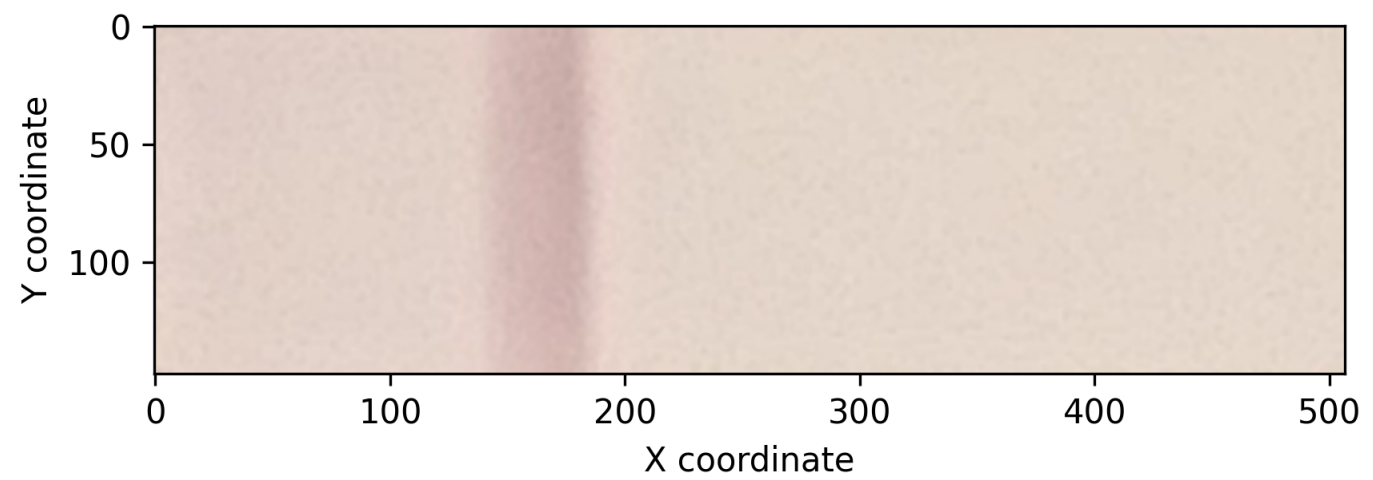

Figure 9. Negative COVID-19 RAT result with only a visible C line.

(a)

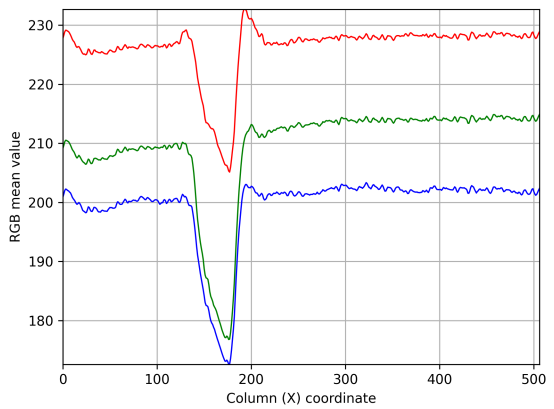

Figure 10. (a) Horizontal RGB profile of a negative COVID-19 RAT, (b) R/G and R/B ratios of the test.

\subsection{Case 3 False negative - weak positive}

COVID-19 RAT was performed by a person at home, and two lines appeared in the test window: $\mathrm{C}$ line and test $(\mathrm{T})$ line. Both lines were visible for at least $20 \mathrm{~min}$ and the test result was determined to be positive. However, after 12 hours, the T line had disappeared from the test window. An image was captured from the aged test window, cropped, and analyzed with rgbxy.py and rgb-iratios.py. The results can be seen in Figure 12, where the second signal is still observable at $x \approx 2000$. The detected signal is at the $T$ line. Therefore the RGB color analysis indicates a positive signal, as well as the original result of the test. 


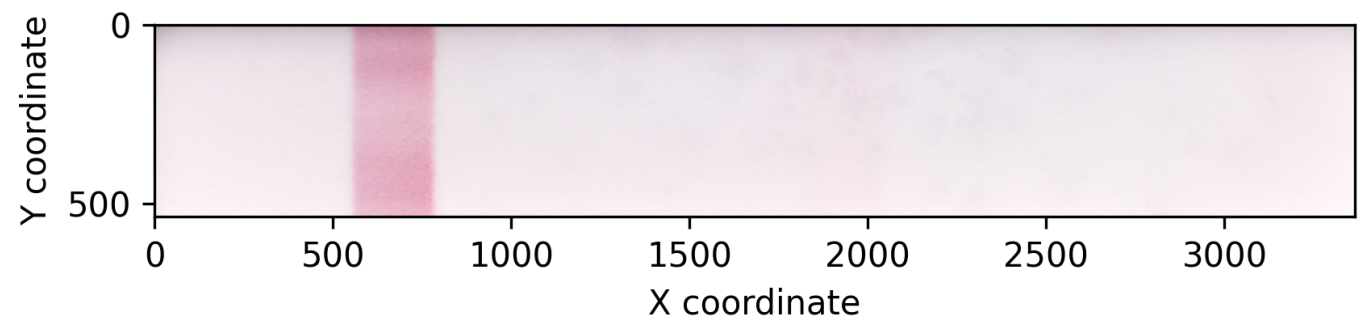

Figure 11. Originally positive COVID-19 RAT turning into negative after several hours (only the C line is visible after 12 hours; however, the $\mathrm{T}$ line was also visible during the first $20 \mathrm{~min}$ test time).

(a)

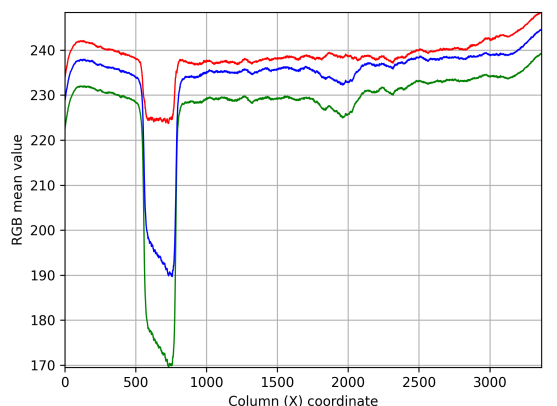

Figure 12. (a) Horizontal RGB profile of a COVID-19 RAT, (b) R/G and R/B ratios of the test.

\subsection{Case 4 Positive}

This COVID-19 RAT gave a positive result, and the test window was captured after 20 min from the start of the test. Both $C$ and $T$ lines are visible in Figure 13. The cropped test window was analyzed, and the strong signals appear at $C$ and $T$ lines. All three figures indicates a positive test result.

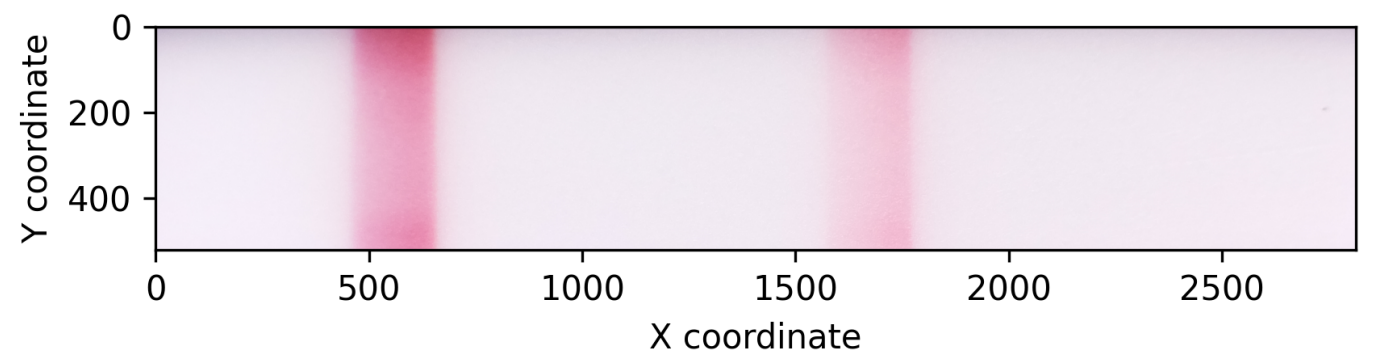

Figure 13. Positive COVID-19 RAT with visible $\mathrm{C}$ and $\mathrm{T}$ lines.

(a)

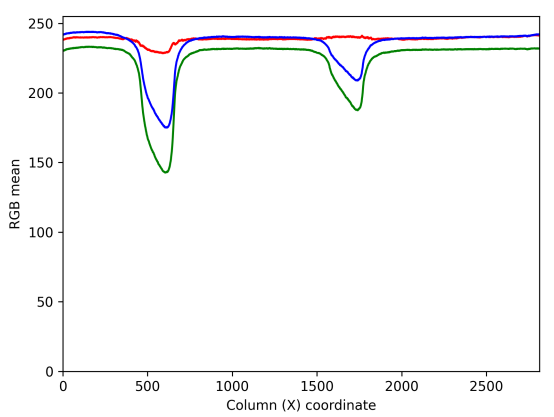

(b)

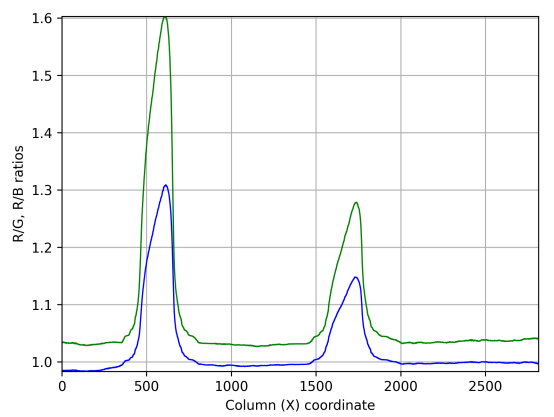

Figure 14. Signals are visible for the $C$ and $T$ lines in (a) the horizontal RGB analysis and (b) the R/G and $\mathrm{R} / \mathrm{B}$ ratios profile. 


\section{Conclusions}

RT-PCR testing is the key criterion, or 'Gold standard test', for identification and hospital discharging for the diagnosed patients. However, repetitive testing leads to increasing costs, jeopardizing the healthcare infrastructure and prolonged hospitalizations [35]. Therefore, alternative test methods have been proposed, out of which RATs emerge as a viable alternative for mass testing. RATs have low cost, short turnaround time, vast availability and ease of use as some of their pros. However, their reported lower sensitivity in comparison to RT-PCR test results require further investigations for identification and early diagnosis [36]. As a contribution to these investigations on the RATs, we proposed an augmented sensitivity method through computer vision, which uses affordable hardware (Raspberry Pi computer and Raspberry Pi camera or mobile phone camera in our case studies) and open source scripts readily available at our Github repository. In these investigations, the principal idea is to use color analysis over the RAT results and detect whether there is any presence of viral antigens and color indication, which may not be seen with the naked eye and, thus, result in false negatives and decrease the sensitivity of RAT kits.

Based on our methodology, we managed to detect false negative results, for which there were no clear indicative $\mathrm{T}$ line on the result window of the test strip. In Case 2, we examined RAT result, for which there was no clear indication of a T line with the naked eye. Thus, the patient commented it as a negative results. However, the next day RT-PCR test result was positive. Our method showed a very weak signal around $\mathrm{T}$ line as seen in Figure 10 (b), which was also interpreted as a positive result. In Case 3, an extraordinary case was assessed such that the color indication around $\mathrm{T}$ line disappeared after 12 hours. Similar to Case 2, our investigations for this case with the color analysis again showed a signal around the $\mathrm{T}$ line, which means that the patient was infected. Therefore, our framework can be used as an inexpensive yet preventive approach, which enhances the current level of sensitivity of RAT kits.

By means of the present framework, sensitivity investigations of samples collected from patients of varying conditions and age groups can be also used to generate statistical models, which provides a deep insight into the transmission routes and risks of the current viral infection. The present framework can be further developed to generate a centralized database and used to provide early diagnosis, or at least preventive actions just in time. Eventually, delayed quarantine of infected individuals and spreading of the current infectious disease can be reduced.

Author Contributions: K.M., J.W., A.K. generated the concept. K.M. was the main programmer and tester while AK assisted for the earlier scripts to realize the work. K.M., L.M., J.W. and A.K. contributed to the writing and editing the final version of the manuscript.

Funding: A.K. gratefully acknowledges the funding from Academy of Finland BESIMAL project (decision number 334197). J.W. also acknowledges the funding from Jenny and Antti Wihuri Foundation.

Institutional Review Board Statement: Not applicable.

Informed Consent Statement: Informed consent was obtained from all subjects involved in the study.

Data Availability Statement: The scripts are accessible at https://github.com/kmiikki/ratcv.

Conflicts of Interest: The authors declare no conflict of interest.

\section{References}

1. Weitzel, T.; Legarraga, P.; Iruretagoyena, M.; Pizarro, G.; Vollrath, V.; Araos, R.; Munita, J.M.; Porte, L. Head-to-head comparison of four antigen-based rapid detection tests for the diagnosis of SARS-CoV-2 in respiratory samples. BioRxiv 2020.

2. Salvagno, G.L.; Gianfilippi, G.; Bragantini, D.; Henry, B.M.; Lippi, G. Clinical assessment of the Roche SARS-CoV-2 rapid antigen test. Diagnosis 2021, 8, 322-326.

3. Porte, L.; Legarraga, P.; Vollrath, V.; Aguilera, X.; Munita, J.M.; Araos, R.; Pizarro, G.; Vial, P.; Iruretagoyena, M.; Dittrich, S.; et al. Evaluation of a novel antigen-based rapid detection test for the diagnosis of SARS-CoV-2 in respiratory samples. International Journal of Infectious Diseases 2020, 99, 328-333. 
4. Sen-Crowe, B.; McKenney, M.; Elkbuli, A. Social distancing during the COVID-19 pandemic: Staying home save lives. The American journal of emergency medicine 2020, 38, 1519-1520.

5. Lippi, G.; Henry, B.M.; Bovo, C.; Sanchis-Gomar, F. Health risks and potential remedies during prolonged lockdowns for coronavirus disease 2019 (COVID-19). Diagnosis 2020, 7, 85-90.

6. Bostanci Ceran, B.; Karakoç, A.; Taciroğlu, E. Airborne pathogen projection during ophthalmic examination. Graefe's Archive for Clinical and Experimental Ophthalmology 2020, 258, 2275-2282.

7. Hobbs, J.E. Food supply chains during the COVID-19 pandemic. Canadian Journal of Agricultural Economics/Revue canadienne d'agroeconomie 2020, 68, 171-176.

8. Pujawan, I.N.; Bah, A.U. Supply chains under COVID-19 disruptions: literature review and research agenda. Supply Chain Forum: An International Journal. Taylor \& Francis, 2021, pp. 1-15.

9. Crozier, A.; Rajan, S.; Buchan, I.; McKee, M. Put to the test: use of rapid testing technologies for covid-19. bmj $2021,372$.

10. Eshghifar, N.; Busheri, A.; Shrestha, R.; Beqaj, S. Evaluation of analytical performance of seven rapid antigen detection kits for detection of SARS-CoV-2 virus. International Journal of General Medicine 2021, 14, 435.

11. Yang, R.; Gui, X.; Xiong, Y. Comparison of clinical characteristics of patients with asymptomatic vs symptomatic coronavirus disease 2019 in Wuhan, China. JAMA network open 2020, 3, e2010182-e2010182.

12. Lavezzo, E.; Franchin, E.; Ciavarella, C.; Cuomo-Dannenburg, G.; Barzon, L.; Del Vecchio, C.; Rossi, L.; Manganelli, R.; Loregian, A.; Navarin, N.; et al. Suppression of a SARS-CoV-2 outbreak in the Italian municipality of Vo'. Nature 2020, 584, 425-429.

13. Khan, S.; Ali, A.; Shi, H.; Siddique, R.; Nabi, G.; Hu, J.; Wang, T.; Dong, M.; Zaman, W.; Han, G.; et al. COVID-19: Clinical aspects and therapeutics responses. Saudi Pharmaceutical Journal 2020, 28, 1004-1008.

14. Burki, T.K. Testing for COVID-19. The Lancet Respiratory Medicine 2020, 8, e63-e64.

15. Nalumansi, A.; Lutalo, T.; Kayiwa, J.; Watera, C.; Balinandi, S.; Kiconco, J.; Nakaseegu, J.; Olara, D.; Odwilo, E.; Serwanga, J.; et al. Field evaluation of the performance of a SARS-CoV-2 antigen rapid diagnostic test in Uganda using nasopharyngeal samples. International Journal of Infectious Diseases 2021, 104, 282-286.

16. Vashist, S.K. In vitro diagnostic assays for COVID-19: recent advances and emerging trends. Diagnostics 2020, 10, 202.

17. Khalid, M.F.; Selvam, K.; Jeffry, A.J.N.; Salmi, M.F.; Najib, M.A.; Norhayati, M.N.; Aziah, I. Performance of Rapid Antigen Tests for COVID-19 Diagnosis: A Systematic Review and Meta-Analysis. Diagnostics 2022, 12, 110.

18. Jääskeläinen, A.; Ahava, M.J.; Jokela, P.; Szirovicza, L.; Pohjala, S.; Vapalahti, O.; Lappalainen, M.; Hepojoki, J.; Kurkela, S. Evaluation of three rapid lateral flow antigen detection tests for the diagnosis of SARS-CoV-2 infection. Journal of Clinical Virology 2021, 137, 104785.

19. Corman, V.M.; Landt, O.; Kaiser, M.; Molenkamp, R.; Meijer, A.; Chu, D.K.; Bleicker, T.; Brünink, S.; Schneider, J.; Schmidt, M.L.; et al. Detection of 2019 novel coronavirus (2019-nCoV) by real-time RT-PCR. Eurosurveillance 2020, 25, 2000045.

20. for Disease Prevention, E.C.; Control. Options for the Use of Rapid Antigen Tests for COVID-19 in the EU/EEA and the UK. Technical Report 2020.

21. Kretzschmar, M.E.; Rozhnova, G.; Bootsma, M.C.; van Boven, M.; van de Wijgert, J.H.; Bonten, M.J. Impact of delays on effectiveness of contact tracing strategies for COVID-19: a modelling study. The Lancet Public Health 2020, 5, e452-e459.

22. Thornton, J. Covid-19: delays in getting tests are keeping doctors from work, health leaders warn. bmj $2020,370, \mathrm{~m} 3755$.

23. Merckx, J.; Wali, R.; Schiller, I.; Caya, C.; Gore, G.C.; Chartrand, C.; Dendukuri, N.; Papenburg, J. Diagnostic accuracy of novel and traditional rapid tests for influenza infection compared with reverse transcriptase polymerase chain reaction: a systematic review and meta-analysis. Annals of internal medicine 2017, 167, 394-409.

24. Pray, I.W. Performance of an antigen-based test for asymptomatic and symptomatic SARS-CoV-2 testing at two university campuses-Wisconsin, September-October 2020. MMWR. Morbidity and mortality weekly report 2021, 69.

25. Embrett, M.; Sim, M. At-home COVID-19 testing: A rapid scoping review 2021.

26. for Disease Control, C.; Prevention.; et al. Interim guidance for antigen testing for SARS-CoV-2 2022.

27. Weitzel, T.; Legarraga, P.; Iruretagoyena, M.; Pizarro, G.; Vollrath, V.; Araos, R.; Munita, J.M.; Porte, L. Comparative evaluation of four rapid SARS-CoV-2 antigen detection tests using universal transport medium. Travel Medicine and Infectious Disease 2021, $39,101942$.

28. Commission, E. COVID-19 in vitro diagnostic devices and test methods database, 2022.

29. Ciotti, M.; Maurici, M.; Pieri, M.; Andreoni, M.; Bernardini, S. Performance of a rapid antigen test in the diagnosis of SARS-CoV-2 infection. Journal of Medical Virology 2021, 93, 2988-2991.

30. Koczula, K.M.; Gallotta, A. Lateral flow assays. Essays in Biochemistry 2016, 60(1), 111-120.

31. Berg, J.M.; Tymoczko, J.L.; Stryer, L. Biochemistry, Fifth Edition: International Version 2002. p. 921-950.

32. for Disease Prevention, E.C.; Control. Options for the use of rapid antigen detection tests for COVID-19 in the EU/EEA - first update. Technical Report 2021.

33. Miikki, K.; Karakoç, A.; Rafiee, M.; Lee, D.W.; Vapaavuori, J.; Tersteegen, J.; Lemetti, L.; Paltakari, J. An open-source camera system for experimental measurements. SoftwareX 2021, 14, 100688.

34. Miikki, K. Raspberry Pi High Speed Camera. https://github.com/kmiikki/rpi-camera, accessed on 2022-02-10.

35. Iancu, R.I.; Zară, A.D.; Mirestean, C.C.; Iancu, D.P.T. Radiomics in COVID-19: The Time for (R) evolution Has Came. BioMed 2022, 2, 60-68. 
36. Mattiuzzi, C.; Henry, B.M.; Lippi, G. Making sense of rapid antigen testing in severe acute respiratory syndrome coronavirus 2 (SARS-CoV-2) diagnostics. Diagnosis 2021, 8, 27-31. 